\title{
Measurement of Sixty-Degree Specular Gloss
}

\author{
By Harry K. Hammond, III, and Isadore Nimeroff
}

\begin{abstract}
Specular gloss is the attribute next to color that is most often used in the evaluation of the appearance of objects. For 10 years the American Society for Testing Materials has used a method of test for the $60^{\circ}$ specular gloss of paint finishes based on research done at this Bureau. The ASTM method prescribes the illuminator and receiver apertures that are to be used. However, in order to determine the uncertainties involved in the calibration of gloss standards, the rate of change of gloss reading with change of aperture was investigated throughout the gloss scale. This was done by modifying an existing goniophotometer so that it became in effect a versatile glossmeter with provision for accurately controlling all of the known geometrical variables involved. Data obtained on the variation of gloss reading with aperture show that standards with widely different distributions of reflected light can be used to check the adjustment of glossmeters and the conformance of their apertures to ASTM specifications in the increasingly important medium- to highgloss range. The problem of obtaining the diffuse correction, which involves source polarization and departure of the specimen from a perfect diffusor, has been investigated.
\end{abstract}

\section{Introduction}

The appearance of an object depends upon several factors; the illuminant, the reflection characteristics of the material, the surface texture, the illuminating and viewing geometry, and the observer. The color and glossiness of a specimen are determined by the spectral composition and geometrical distribution of the incident light and upon the transformations that take place upon reflection from the specimen. These two appearance attributes, color and gloss, are somewhat related. Color measurement may require correction for surface glossiness. Specular gloss measurement may require correction for diffuse reflectance.

The complete description of the light-reflecting properties of a specimen would require an infinite number of measurements of reflectance throughout a hemisphere for an infinite number of directions of illumination. Even an approach to such a set of data is uneconomical to obtain with present goniophotometric equipment. Usually one must be content to obtain one goniophotometric reflection curve for a single direction of illumination (see fig. 1). Furthermore, the question of how to interpret a goniophotometric curve still remains, for in contrast to spectrophotometric curves there

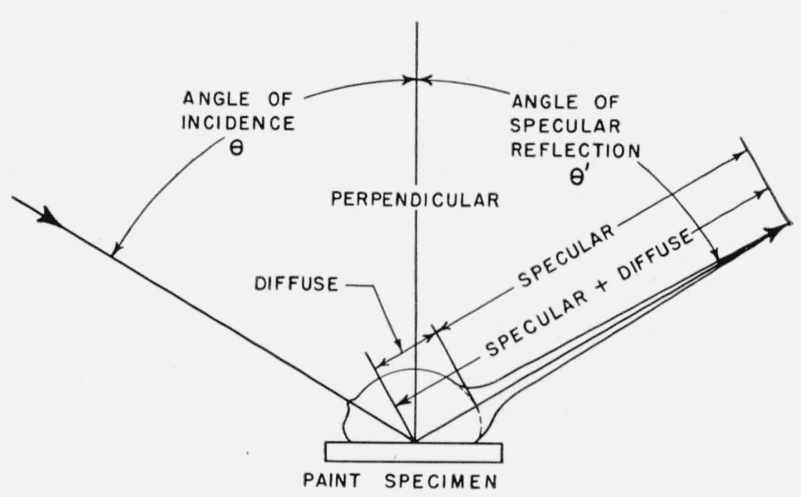

Figure 1. Typical goniophotometric curve for a paint specimen, with defining terms.

has not yet been developed a satisfactory method for reducing the former to a simple meaningful numeric or set of numerics as has been done for the latter. Because of these circumstances, one must generally be content to measure the specular reflectance for one direction of illumination. Previous investigations have shown that the choice of direction as well as aperture for illumination and view is governed by the type of material involved. Hunter and Judd $[1]^{1}$ found that $60^{\circ}$ specular gloss correlated most satisfactorily with

${ }_{1}^{1}$ Figures in brackets indicate the literature references at the end of this paper. 
the surface appearance of paint finishes, and a method based on their investigations at this Bureau was tentatively adopted by ASTM in 1939. In order to simplify the discussions in this paper the problems treated are confined to $60^{\circ}$ specular gloss, but the considerations involved apply to other specular angles as well.

Specular gloss could be measured without instrumental complication if there existed (1) an infinitesimal, high intensity, unpolarized light source; (2) perfect collimators; and (3) an infinitesimal but sensitive receiver. Then glossmeters could be readily duplicated. Practical sources, collimators, and receivers cause difficulty because imperfections in their construction combine with differences in reflected-light-flux distributions to give inconsistent gloss readings for various types of materials. If it were practical to make glossmeters with identical geometrical features, there would be no difficulty in comparing measurements made on different instruments. A more practical approach to the problem, however, lies in further standardizing gloss measurement by specifying tolerances for glossmeter geometry.

The original investigation, already referred to, on which the ASTM method is based, employed rectangular source and receiver apertures. This was done for two reasons. First, the type of lamp employed in the apparatus had a single, coiled filament producing a long narrow source, the cross section of which is rectangular; second, if the coiled lamp-filament were oriented with its axis perpendicular to the plane of measurement (see definition below) more light would be available because a larger aperture could be permitted in a plane perpendicular to the plane of measurement without appreciably affecting the gloss reading. Similarly, a proportionately larger receiver aperture in the plane perpendicular to the plane of measurement permits the receiver to gather light that would otherwise be lost because of change in direction on reflection from brush marks on brushed paint panels, when these are oriented, as they should be, with the marks parallel to the plane of measurement.

In order to determine the permissible geometrical tolerances, a "versatile" glossmeter has been developed. This instrument has provision for making the following adjustments:
1. The receiver aperture may be varied either parallel to or perpendicular to the plane of measurement.

2. The source aperture may be varied either parallel to or perpendicular to the plane of measurement.

3. The position of the image of the source may be varied with respect to the plane of the receiver entrance window.

\section{The specular angle may be varied.}

In addition to determining the permissible geometrical tolerances, there remains the problem of obtaining the diffuse correction. Measured values of diffuse correction do not agree exactly with those obtained by simple theory; however, the small discrepancy is accounted for by (1) The departure of the reflected light distribution of the specimen from that of the perfect diffusor; (2) The degree of polarization of the source; and (3) The reflectance of the surface.

\section{Terms, Symbols, and Definitions}

The purpose of glossimetry is to evaluate objectively some variable that may be used as an index of appearance ascribable to gloss. The most conveniently used variable involves some function of the geometrical distributions of the reflected light. For this reason it is advisable to define common reflectance terms as well as the more specialized concepts associated primarily with gloss measurement.

In addition to the definitions given below, there are two general concepts that should be discussed. The adjective "luminous" should be used whenever it is important that the defined term include weighting of the spectral energy distribution according to the luminosity function. A distinction must also be drawn between what may be called surface reflectance and body reflectance. Reflection of the latter type may be described as reemission when the light penetrates the surface of the specimen and re-emerges at the incident face with a geometric distribution similar to that of a self-luminous source. The definitions of specular reflectance and diffuse reflectance given below involve the concepts of surface reflectance and body reflectance (re-emission), respectively. 
The most important terms, definitions, and symbols used in this paper are given below.

Illuminance. Luminous flux incident per unit area of surface.

Luminous intensity (of reflected luminous flux in any direction). The solid-angular flux density.

Luminance. Luminous flux emitted per unit solid angle and per unit projected area.

Luminous (total) reflection. A general term for the process by which a part of the incident luminous flux leaves a specimen from the incident side.

Specular angle, $\theta^{\prime}$. The angle between the perpendicular to a plane surface and the reflected ray. This angle is numerically equal to the angle of incidence, $\theta$, and lies in the same plane on the opposite side of the perpendicular; it is often designated $-\theta$ (refer to fig. 1).

Luminous specular reflection. The process by which incident luminous flux is reflected from a surface in an image-forming state.

Luminous diffuse reflection. The process by which incident luminous flux is reemitted (in a nonimage-forming state).

Luminous specular reflectance. Ratio of luminous flux leaving a surface in an image-forming state to the incident luminous flux.

Luminous diffuse reflectance. Ratio of luminous flux reemitted at the illuminated surface of a nonself-luminous specimen to the incident luminous flux.

Luminous (total) reflectance. Ratio of luminous flux reflected by a specimen to the luminous flux incident on it. Diffuse reflectance plus specular reflectance equals total reflectance. It should be noted that the specular and diffuse reflectance components often cannot be separated for measurement.

Luminous directional reflectance. Ratio of luminous flux from the specimen when illuminated and viewed from specified directions to that from the perfectly reflecting, perfectly diffusing specimen, similarly illuminated and viewed.

Luminous fractional reflectance of a specimen. Ratio of luminous flux reflected within a specified solid angle to the luminous flux incident on the specimen.

Goniophotometer. An instrument used to obtain the geometric distribution of luminous flux.
Goniophotometric reflection curve. A plot of the geometric distribution of reflected luminous flux, for a specified distribution of incident flux, as a function of angle of view (see fig. 1).

Gloss. The function of luminous directional reflectance of a specimen responsible for its shiny or lustrous appearance.

Specular gloss, $G_{s}$. The ratio of the specularly reflected luminous flux, $F_{x}$, from a specimen surface for a specified angle of incidence, $\theta$, to the specularly reflected luminous flux, $F_{s}$, from a standard surface for the same angle of incidence. Written symbolically:

$$
G_{s}=\left(F_{x} / F_{s}\right)_{\theta, \theta^{\prime}}
$$

where $\theta^{\prime}$ is the specular angle.

Specular glossmeter. An instrument for measuring specular gloss at a fixed specular angle.

Geometric conditions of illumination and view. Include the specular angle and the apertures of the source and receiver.

Source. A luminous body or area by which the specimen is illuminated.

Receiver. A photocell or an enclosure equipped with photocells responding to luminous flux from the specimen. The sensitive surface of the photocell or the opening to the enclosure is called the receiver entrance window.

Plane of measurement. The plane passing through the axes of incidence and view.

Aperture (of a source or receiver). The plane angle subtended by the source or receiver entrance window at the respective collimating lenses. With rectangular apertures the angles, $\alpha$ and $\beta$, may be used to describe the angular size of the aperture in the plane and perpendicular to the plane of measurement, respectively. In computing an aperture it is convenient to find the tangent of the half angle of the aperture. For example: the source aperture, $\alpha$, in the plane of measurement is equal to $2 \tan ^{-1}(y / f)$, where $y$ is the half dimension of the source in the plane of measurement, and $f$ is the focal length of the collimating lens.

Fresnel reflector (nonmetallic). Specularly reflects a fraction, $G_{s}$, of luminous flux computed from the Fresnel equation [2],

$$
G_{s}=\frac{E}{2}\left[\frac{\sin ^{2}(i-r)}{\sin ^{2}(i+r)}+\frac{\tan ^{2}(i-r)}{\tan ^{2}(i+r)}\right],
$$


where $E$ is the illuminance, $i$ is the angle of incidence, and $r$ is the angle of refraction.

A more convenient form of this equation is obtained by expressing the angle of refraction in terms of the angle of incidence and the index of refraction, $n$, of the material. This is:

$G_{s}=\frac{E}{2}\left\{\frac{\cos i-\sqrt{n^{2}-\sin ^{2} i}}{\cos i+\sqrt{n^{2}-\sin ^{2} i}}+\frac{n^{2} \cos i-\sqrt{n^{2}-\sin ^{2} i}}{n^{2} \cos i+\sqrt{n^{2}-\sin ^{2} i}}\right\}$,

Perfect diffusor. Reflects light according to Lambert's Cosine Law, which states that the solid angular density of the luminous flux reflected from the surface in any direction varies as the cosine of the angle between that direction and the perpendicular to the surface.

Glossiness. The lustrous appearance of a surface ascribable to its gloss.

Lightness. That appearance attribute of a specimen which correlates with its luminous diffuse reflectance.

\section{Glossmeters}

Instruments for the measurement of specular gloss have been used for more than 30 years. In 1937, however, Hunter [3] described a photoelectric modification of the McNicholas goniophotometer, which is the earliest known photoelectric specular glossmeter. Since then glossmeters of this type and similar photoelectric types have been manufactured commercially.

\section{Theoretical Glossmeter}

The theoretical glossmeter consists of a source, source and receiver collimators, and a receiver (see fig. 2). If we had to deal only with an infinitesimal, unpolarized light source of high intensity, an infinitesimal receiver of high response, and perfect collimators, the problem of geometric tolerance specification would be eliminated. Such hypothetical glossmeter elements would have minimum aperatures and no lens aberrations and could be duplicated without causing error in the gloss readings. Measurement of specular gloss would then be completely reproducible from instrument to instrument if the specular angles were accurately determined.

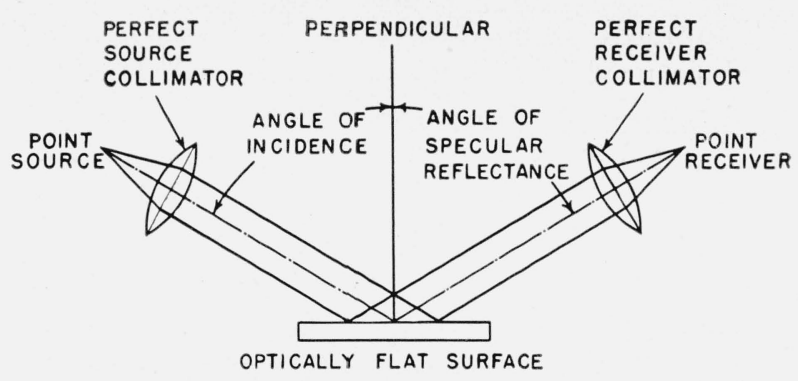

FIGURE 2. Theoretical glossmeter, employing point source and point receiver.

\section{Practical Glossmeters}

Most glossmeters in commercial use today employ an incandescent-filament lamp as a light source, a source lens to collimate the incident beam, a receiver lens to obtain at the receiver entrance window an image of the source reflected from a Fresnel reflector, and some type of photoelectric receiver [4], (see fig. 3). The source aperture is

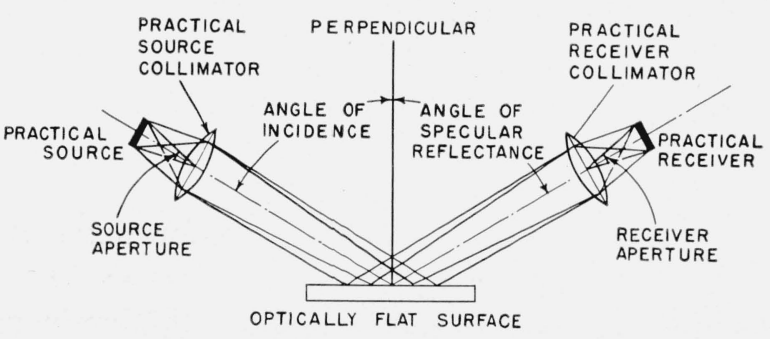

Figure 3. Practical glossmeter, employing practical source and practical receiver.

determined by the dimensions of the source and the focal length of the collimating lens. The receiver aperture is determined in a similar manner, using the dimensions of the receiver entrance window. Standard apertures may be specified, but errors of duplication cannot be avoided.

The collimating lenses used in commercial glossmeters are usually simple, uncorrected lenses, subject to such lens errors as astigmatism, spherical and chromatic aberration, and coma. Corrected lenses could be used, but the accuracy required in most gloss measurements can be obtained without this added expense. Because most commercial glossmeters are compactly made, the distances between elements and the apertures are physically small. This leads to errors in the setting of the specular angle, measurement of the source and receiver apertures, collimation of the incident beam, and positioning of the focussed im- 
age of the source in the center of the receiver entrance window when reflected from a flat mirror surface.

\section{The Versatile Glossmeter}

In order to investigate the precision to which the many geometric variables of a glossmeter must be specified, an existing goniophotometer was modified to make a versatile glossmeter (see fig. 4). This glossmeter has provision for accurately controlling (1) the source and receiver apertures in both the $\alpha$ and $\beta$ planes; (2) the angles of illumination and view; (3) the collimation; and (4) the position of the source image relative to the plane of the receiver aperture. Each of these variables can be changed individually to any desired degree of departure from the ASTM specifications.

Control of the source aperture is accomplished by employing a pair of condensing lenses to focus an image of the source on an adjustable metallic aperture. This aperture, serving as the new source of controllable size, is located in the focal plane of an achromatic collimating lens, the focal length of which is accurately known.

The specular angle is easily controlled since the source and receiver are located on the hori ontal arms of a goniophotometer of which the angular scale may be set to within $\pm 0.1^{\circ}$, and the specimen of which the gloss is to be measured is placed vertically on the turntable of the goniophotometer.

The receiver collimating lens also is an achromatic lens for which the focal length is accurately known. It is of such aperture and so positioned that there is no vignetting of rays that should reach the receiver. The receiver aperture is precisely located so that the image of the source is brought to focus at the plane of this aperture. Since there is no need to conserve space, this glossmeter was made large enough to allow for high accuracy of measurement of the apertures and the distances between elements.

\section{Glossmeter Variables}

A surface lacking the smoothness of polished black glass will reflect some of the incident light in directions adjacent to that of specular reflection; consequently, some of the reflected light may fail to reach the instrument receiver, and a lower gloss reading may be obtained. It should

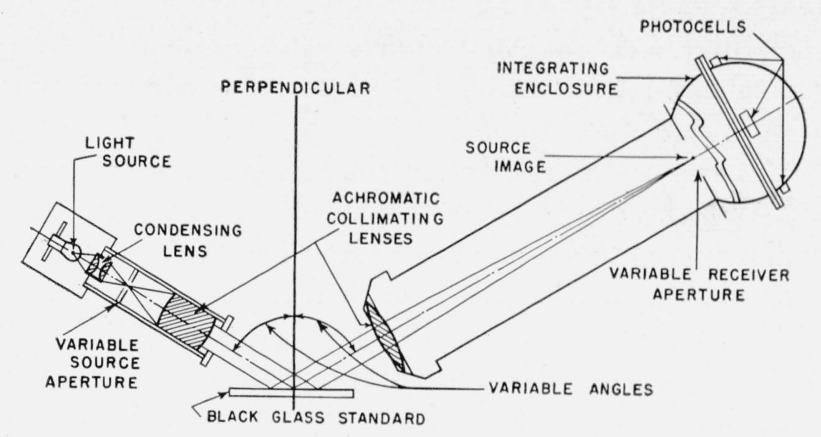

Figure 4. NBS versatile glossmeter, employing variable source and receiver apertures, achromatic collimators, and variable angles of illumination and view.

be pointed out that the specular reflectance, and consequently the gloss, of a nonmetallic specimen is a function of the index of refraction of the material of the specimen (see eq 3) as well as of the smoothness of the surface. Polished black glass of known index of refraction has been used to calibrate the upper end of the gloss scale. For low-gloss finishes, such as are used for camouflage by the Armed Services, the plane surface of a block of magnesium carbonate, which approximates the perfect diffuser, has been used as a standard at the lower end of the scale. This leaves the question of what material to use for intermediate standards. Horning and Morse [5] implied that in the medium- to low-gloss range average paint specimens have reflected lightflux distributions more like glazed ceramic tiles than like depolished black glass. From this standpoint tile should be preferable for use as gloss standards in the paint industry.

Recent cooperative work with the SherwinWilliams Co. revealed, however, that better agreement in the measurement of high-gloss paints had been obtained on different glossmeters with the use of depolished black glass standards than with ceramic tile standards. In fact ceramic tile standards produced much larger instrument discrepancies for the same specimens. In order to analyze this situation, goniophotometric curves of fractional reflectance in the region of specular reflection were obtained for the paint specimen, a tile standard, and a sand-blasted depolished black glass standard having about the same $60^{\circ}$ specular gloss (80 on the ASTM scale). These curves are shown in figure 5. For these measurements the goniophotometer source aperture was made $0.3^{\circ}$ circular, and the receiver aperture was made $0.6^{\circ}$ 
circular. A polished black glass standard was used, and the results are plotted on a fractional reflectance scale times 1,000 , thus giving a value of 1,000 to the perfectly reflecting plane surface. From these curves it is seen that the light-flux distribution of the sand-blasted black glass standard is very similar to that of this particular highgloss paint specimen, whereas the distribution of the tile standard is quite different. If a glossmeter having accurate ASTM apertures is used to determine the gloss of this paint specimen, the reading will be the same no matter which standard is used. On the other hand, if the receiver aperture were too large, very nearly the true gloss will be obtained with the glass standard, but a much lower reading will be obtained for this specimen if the tile standard is used for calibrating the instrument. The conclusion might be drawn at this point that instrumental aperture variation might be minimized by using the glass standard, and this would be true if all high-gloss specimens had flux distributions like the one illustrated here. However, as implied by Horning and Morse, many specimens are encountered with reflected light-flux distributions more like that of the tile standard, in which case nearly correct gloss readings would be obtained with this standard even though the receiver aperture were too large, whereas calibration with the glass standard would cause the instrument to give a reading higher than the correct value. Thus it is seen that if the reflected light-flux distributions of specimen and standard are nearly identical, the size of the apertures is not critical.

However, it is not practical to determine the distribution of each type of specimen, and it is even less practical to attempt to duplicate these distributions even approximately with durable materials that might be used as standards. The more practical approach to the problem is to make the glossmeter apertures as accurate as possible so that the instrument will give correct gloss readings no matter what the reflected light distribution of the specimen. Although glossmeter manufacturers may claim that the apertures are exactly correct, it would be desirable for the instrument operator to have a simple method to check their accuracy. This may be done by taking advantage of the facts pointed out above, namely that a glossmeter will read correctly two standards hav-

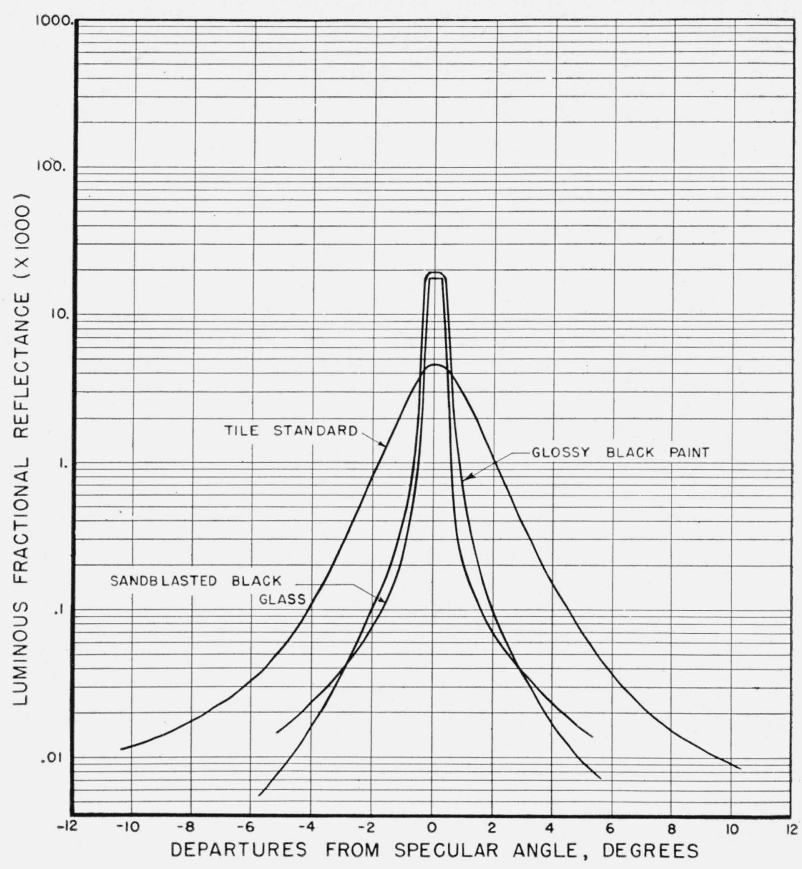

FiguRE 5. Luminous fractional reflectance curves of highgloss materials.

Angle of illumination and view, $60^{\circ}$, source aperture, $0.3^{\circ}$, circular, receiver aperture $0.6^{\circ}$.

ing radically different distributions of reflected light-flux only when apertures are correct.

In the usual method of glossmeter operation, a polished glass surface is employed to make the initial calibration. The reading subsequently obtained for a tile standard should be the true value if the apertures are correct, too large if the receiver aperture is too large or too small if the aperture is too small, other adjustments being correct. It may be noted that the reproducible plane surfaces of different refractive index employed by Moore and Hunter [6] to avoid inaccuracies resulting from abrasions of a black glass standard do not serve to disclose errors arising from nonstandard apertures. In contrast to reflectance measurements where the apertures of the illuminating and viewing beams are relatively unimportant, the apertures employed in gloss measurement must be carefully specified and held with close tolerance if accurate glossimetry is to be accomplished. It should be stated here that in contrast to previous practice, it has been found not sufficient to specify the combined spreads of source and receiver aperture but each must be specified separately because 
the ratio of source aperture to receiver'aperture affects significantly the measured gloss value.

\section{Receiver Apperture}

To illustrate the magnitude of the effect of variation of the rectangular receiver aperture in the plane in which the specular angle is measured, designated $\alpha$, and in the plane in the polar direction perpendicular to $\alpha$, designated $\beta$, two series of measurement were made on plaques of different gloss. This was done for both depolished (sandblasted) black glass, and for glazed ceramic tiles. The results of these measurements are illustrated graphically in figures 6 and 7 . These figures show the effect of variation in receiver aperture in the $\alpha$ and $\beta$ planes, respectively, on the measured gloss values of tile and depolished black glass standards. It will be noted that the measured values for the tiles are a pronounced function of

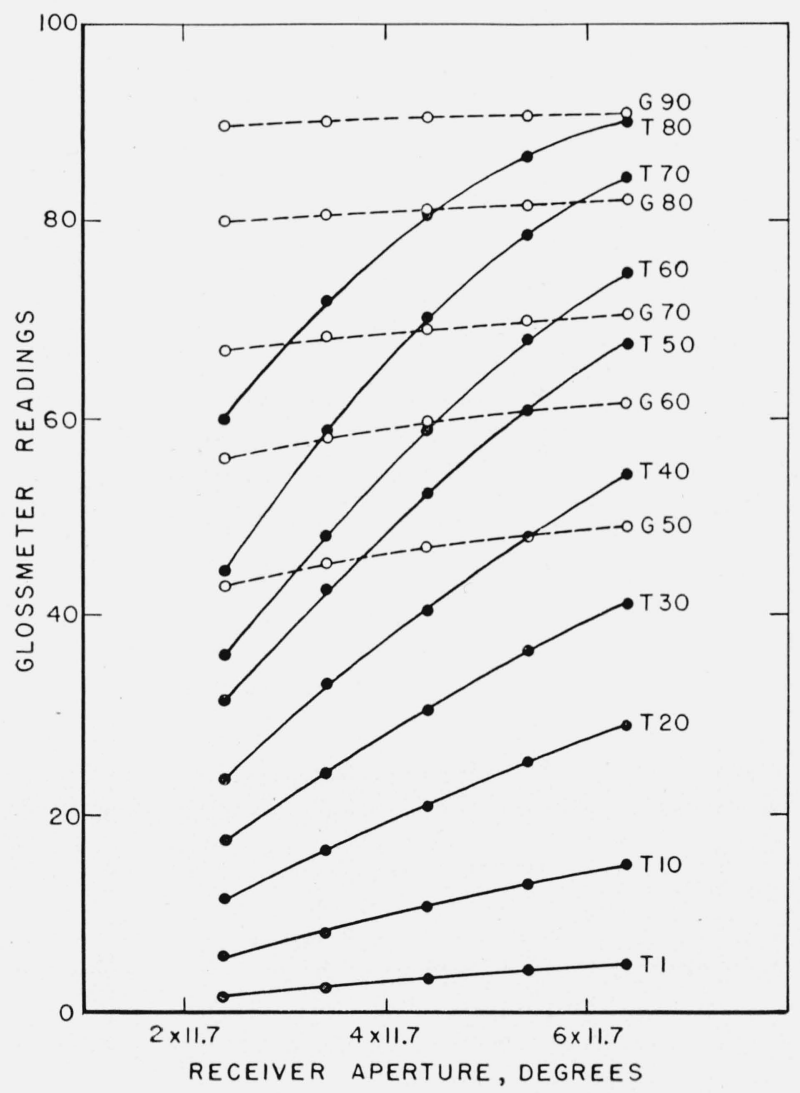

FIGURE 6. Glossmeter readings obtained by varying the dimension of the receiver aperture in the $\alpha$ plane.

The depolished black specimens are designated $G$, followed by the nominal gloss value; the glazed ceramic tile specimens are designated $T$, followed by the nominal gloss value. the receiver aperture. On the other hand, the measured values for the depolished black glass standards remain reasonably constant over the wide range of apertures employed. This is ascribable to the fact that high-gloss tile produces narrow-angle scattering near the specular angle, whereas depolished black glass produces wideangle scattering.

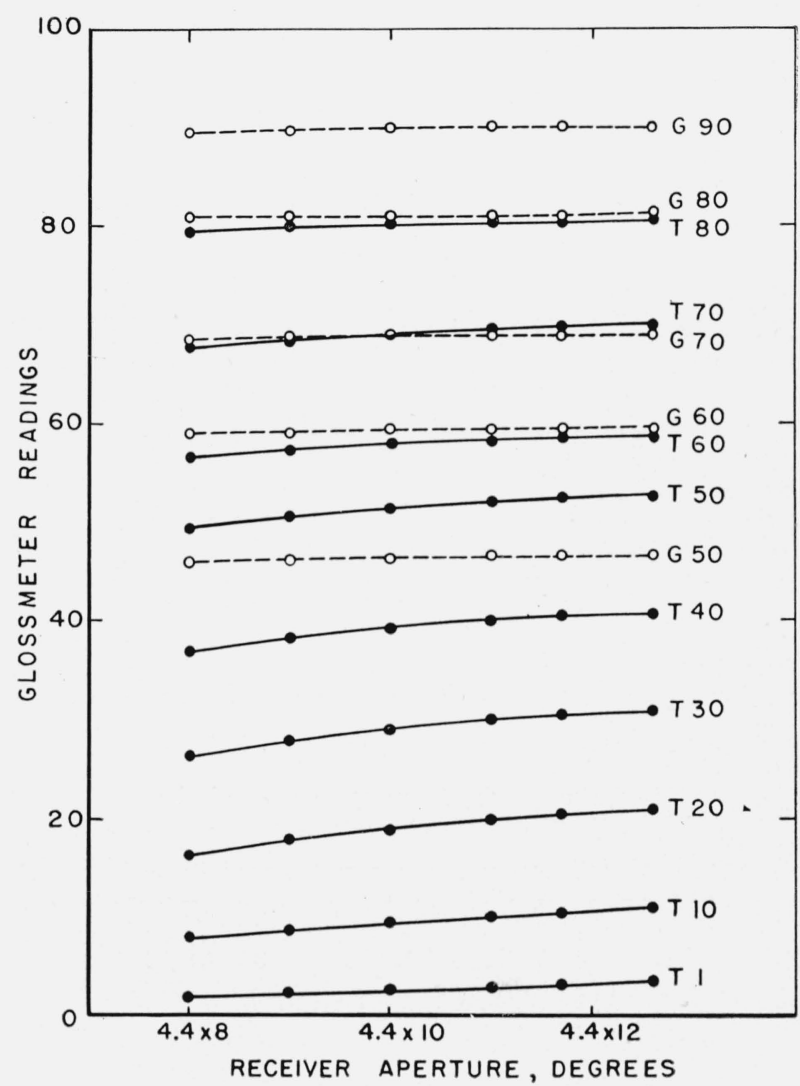

FIGURE 7. Glossmeter readings obtained by varying the dimension of the receiver aperture in the $\beta$ plane.

The depolished black glass specimens are designated $G$, followed by the nominal gloss value; the glazed ceramic tile specimens are designated $T$, followed by the nominal gloss value.

\section{Source Aperture}

To determine the effect of source aperture variation on glossmeter readings and thus be able to determine permissible tolerances, data were obtained on the variation of measured gloss with source aperture with the receiver aperture held constant at the value prescribed in the ASTM method mentioned earlier. It was found that the variation of source aperture in the $\alpha$ plane affected the measured gloss of the plaques as shown in 
figure 8; variation in this plane did not produce as pronounced measured gloss differences as for the receiver aperture variation. Variation in the $\beta$ plane had a negligible effect on the measured gloss values, so the data are not shown here.

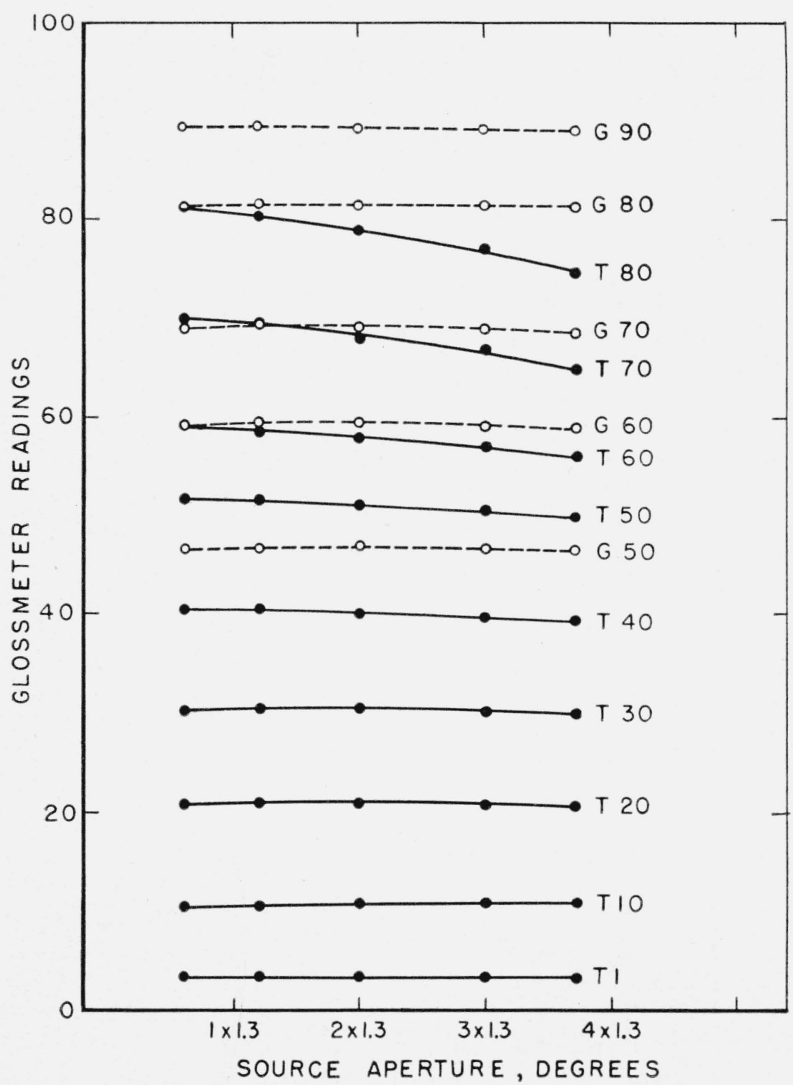

FIGURE 8 . Glossmeter readings obtained by varying the dimension of the source aperture in the $\alpha$ plane.

The depolished black glass specimens are designated $c$, followed by the nominal gloss value; the glazed ceramic tile specimens are designated $T$, followed by the nominal gloss value.

\section{Position of Source Image}

Another question of practical importance is the effect of the position of the image of the source. A properly adjusted glossmeter will form an image of the source centered in the receiver entrance window when a flat polished surface is placed in the specimen position. Commercial glossmeters using simple lenses are difficult to adjust because chromatic abberration of the lenses causes light of different wavelengths to focus at different positions. If the red rays are brought to a sharp focus, the source image is surrounded by a blue haze. Conversely, if the blue rays are brought to a sharp focus, the image will be surrounded by a red haze. The effect of position of the source aperture with respect to the receiver entrance window was explored on the experimental glossmeter by placing spectacle-type lenses of low power between the specimen and receiver collimating lens. This procedure was used because the collimating lenses are not easily moved from their positions of optimum collimation. By using positive or negative lenses the image of the source was obtained before or behind the plane of the receiver entrance window. Figure 9 illustrates the effect produced on the measured gloss values. It shows that the greatest effect is produced on the glass values of the tile plaques, and that the effect on measured gloss values is not negligible for tile but is practically so for depolished glass. The data also show that there is no preferred direction of

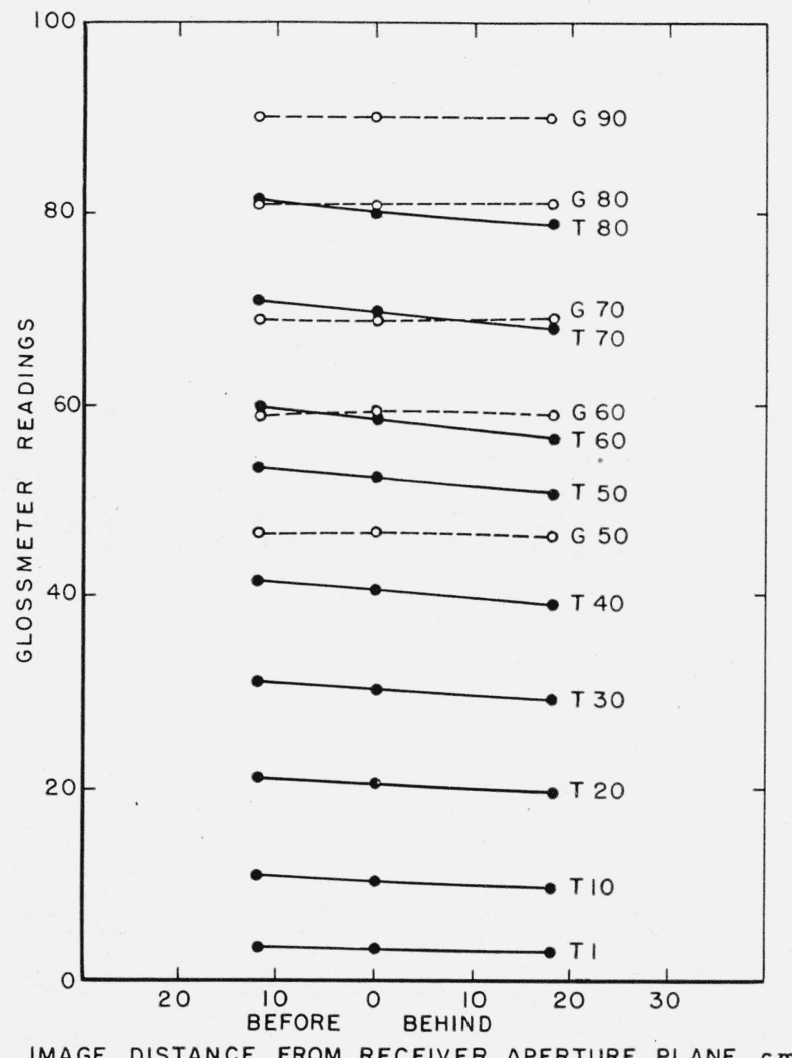

FIGURE 9. Glossmeter readings obtained by varying the position of the focused image of the source relative to plane of the receiver aperture.

The depolished black glass specimens are designated $G$, followed by the nominal gloss value; the glazed ceramic tile specimens are designated $T$, followed by the nominal gloss value. Focal length of receiver lens is $54.6 \mathrm{~cm}$. 
departure from the plane of the receiver entrance window.

\section{Specular Angle}

Glossmeters are usually adjusted so that the image of the source reflected from a plane reflector, such as that of polished opaque glass, falls on the center of the receiver entrance window. Suppose, however, that in making this adjustment the angle of incidence is disturbed and that it is found to be $59^{\circ}$ or $61^{\circ}$ instead of $60^{\circ}$. What effect will this error in the specular angle have on the gloss readings? It can be shown from the Fresnel equation (see definition of Fresnel reflector) that a departure of $1^{\circ}$ in specular angle at $60^{\circ}$ will cause a change of approximately 5 percent in the specularly reflected flux from a polished surface of refractive index 1.53. In the usual method of operation, the instrument is set to read correctly the value of the working standard, then the gloss of the test specimen is read relative to it. If the standard and specimen have nearly the same sur-face characteristics, no appreciable error will result.

Figure 10 shows the effect on the measured gloss of both tile and depolished glass standards with departure from the $60^{\circ}$ specular angle. The illuminance was adjusted for each angle so that the instrument indicated the $60^{\circ}$ specular gloss of the polished glass reference standard. It will be noted from the figure that for the range of angles investigated the measured gloss of the depolished glass plaques increases with increasing specular angle, but the measured gloss of the tile plaques decreases slightly. In making the adjustment for the standard, the illuminance for $59^{\circ}$, as an example, had to be increased. Then two factors are operating, (1) the increased illuminance and (2) the decreased directional reflectance due to Fresnel reflection. The effect on the depolished glass plaques is explainable by the assumption that the second factor acts more importantly than the first, since the surface is composed of many facets in the gross plane of the plaque. The effect on the tile plaques is explainable by the assumption that the second factor is operating less importantly than the first, since the higher index of refraction of this material causes a smaller relative increase in reflectance with increasing specular angle. Since the instrument is caused to give the same reading for the reference standard the depolished black glass plaques show slight increase in relative gloss, whereas the tile plaques show slight decrease in relative gloss with increasing angle of incidence.

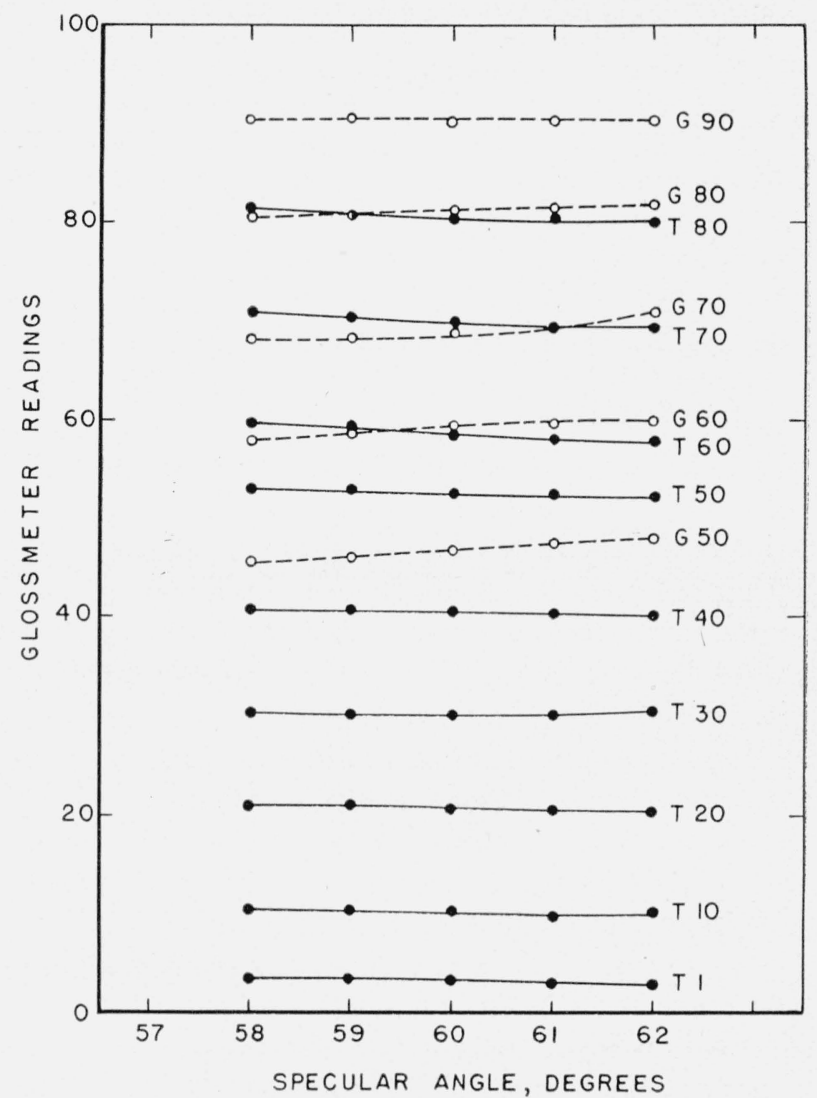

Figure 10. Glossmeter readings obtained by varying the specular angle.

The depolished black glass specimens are designated $c$, followed by the nominal gloss value; the glazed ceramic tule specimens are designated $T$, followed by the nominal gloss value.

\section{Diffuse Correction}

\section{Simple Theory}

We come now to the problem of diffuse correction. Figure 1 shows that the reflected light received by the glossmeter may be considered to be composed of two parts, the specular reflection or true gloss contributed by the surface of the specimen and the diffuse reflection contributed by the body of the specimen. Thus we see that the lightscattering property of the body of the specimen, or in a sense its body color, affects the measured gloss. In the case of high-gloss specimens, the diffuse component is a small fraction of the instrument reading, and many laboratories working with high-gloss materials do not correct or allow for it. 
In the case of low-gloss specimens, however, this correction should be made especially when comparing specimens of different lightness.

\section{(a) Hemisphere Method}

Picture a perfectly reflecting, perfectly diffusing specimen which reflects no light specularly but which causes a "gloss" reading on the instrument because some of the diffusely reflected light reaches the receiver. What should be the magnitude of the correction to be substracted from the instrument reading to obtain the true gloss of the specimen? Lambert [7] showed that a perfectly diffusing specimen reflects light in such a manner that the luminous intensity in any direction is proportional to the cosine of the angle, $\psi$, between the perpendicular to the surface and the direction of reflection. He also showed that the perfect diffuser would appear equally bright in all directions, that is, that the luminance is constant.

Let us assume that the luminous flux reemitted by a perfect diffusor having luminance, $B$, and area, $d A$ falls normally on the surface of a hemisphere of radius, $R$ (see fig. 11). First we wish to compute the total flux, $F$, falling upon the entire hemisphere. The flux, $d F$, in a direction making an angle $\psi$ with the perpendicular to the diffusor is evidently proportional to the projection of $d A$ on a plane perpendicular to the direction $\psi$, or, $d A \cos \psi$, and to the solid angle, $d \Omega$, subtended at the diffusor. The proportionality constant is the luminance, $B$. Thus

$$
d F=B \cos \psi d A d \Omega .
$$

The solid angle,

$$
d \Omega=2 \pi R^{2} \sin \psi d \psi / R^{2}=2 \pi \sin \psi d \psi,
$$

where $d \Omega$ is now the hollow cone subtended at the specimen by the zone of width $R d \psi$, whose semiangle is $\psi$. Therefore,

$$
F=\pi B d A \int_{0}^{\pi / 2} 2 \cos \psi \sin \psi d \psi=\pi B d A .
$$

Now consider the flux, $f$, which falls on the receiver at an angle $\theta^{\prime}$.

From eq 4 above

$$
d f=B \cos \theta^{\prime} d A d \omega .
$$

The solid angle subtended by this receiver is

$$
d \omega=\frac{R^{2} \alpha \beta}{R^{2}}=\alpha \beta
$$

where $\alpha$ is the aperture angle in the plane of measurement (the plane in which the specular angle $\theta^{\prime}$ is measured), and $\beta$ is the aperture angle in the plane perpendicular to the plane of measurement. The angles $\alpha$ and $\beta$ are small and are measured in radians. Then

$$
f=B \alpha \beta \cos \theta^{\prime} d A .
$$

Thus, of the reemitted luminous flux, the fraction, $F_{r}$, entering the receiver aperture is

$$
F_{r}=\frac{f}{F}=\frac{\alpha \beta \cos \theta^{\prime}}{\pi} .
$$

For the ASTM receiver,

$$
\alpha=4.4^{\circ}, \beta=11.7^{\circ} \text {, and } \theta^{\prime}=60^{\circ} \text {. }
$$

Thus $F_{r}$ is computed to be 0.0025 . This means that the perfectly reflecting, perfectly diffusing specimen although in reality having no specular gloss would cause the instrument to read 2.5 if a perfect plane reflector were to read 1,000.

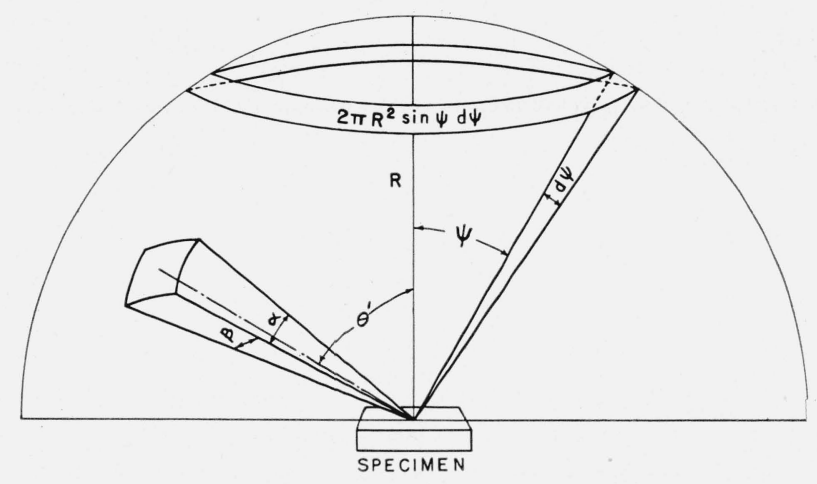

Figure 11. Diffuse correction for the perfectly diffusing surface treated by the hemisphere method on the basis of simple theory.

(b) Tangent Sphere Method

The magnitude of the diffuse correction may be obtained very simply by considering the illuminance $E$ at any point on a sphere of diameter $D$ tangent to the diffusor (see fig. 12). Let the 


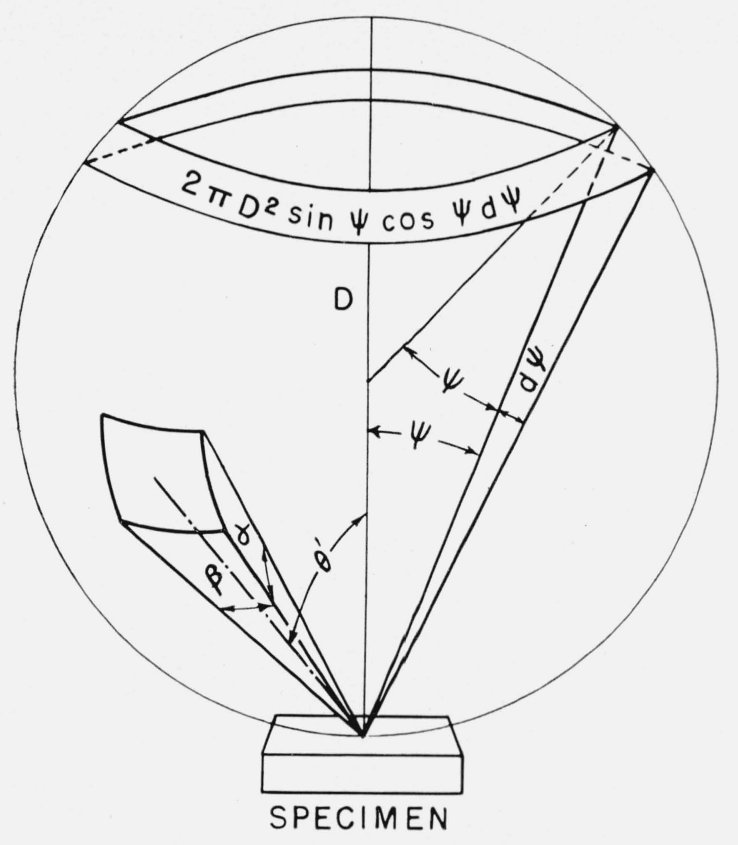

Figure 12. Diffuse correction for the perfectly diffusing surface treated by the tangent sphere method on the basis of simple theory.

luminous intensity in any direction perpendicular to the perfect diffusor of area $d A$ be represented by $I_{0} d A$ and the intensity in other directions by $I_{\psi} d A$. Then,

$$
I_{\psi} d A=I_{0} \cos \psi d A
$$

The illuminance, $E_{\psi}$, at any point on the sphere is the intensity in that direction multiplied by the cosine of the angle of incidence and divided by the square of the distance, $D^{2} \cos ^{2} \psi$, from the perfect diffusor to that point on the sphere. Therefore,

$$
E_{\psi}=\frac{I_{\psi} \cos \psi d A}{D^{2} \cos ^{2} \psi}=\frac{I_{0} d A}{D^{2}} .
$$

Thus we see that the illuminance on the sphere is independent of the angle of view. Therefore the ratio, $F_{r}$, of the flux on a receiver to the total flux is simply the ratio of the area, $a$, of the receiver projected on the sphere to the area, $A^{\prime}$, of the whole sphere. But $a$, located at an angle $\theta^{\prime}$ from the perpendicular to the diffusor, is $\alpha \beta D^{2} \cos \theta^{\prime}$ and $A^{\prime}$ is $\pi D^{2}$. Therefore,

$$
F_{r}=\frac{a}{A^{\prime}}=\frac{\alpha \beta D^{2} \cos \theta^{\prime}}{\pi D^{2}}=\frac{\alpha \beta \cos \theta^{\prime}}{\pi} .
$$

This is the same as eq 7 obtained by integration of luminous flux over the hemisphere surface. It should be noted that the diffuse correction is the ratio of the area of the tangent sphere intercepted by $\alpha$ and $\beta$ to the area of the whole sphere. It may be said, therefore, that the diffuse correction is numerically equal to the fraction of the tangent sphere intercepted by the solid angle formed by the instrument aperture.

\section{Expansion of Theory}

Practical surfaces, neither perfectly reflecting nor perfectly diffusing, will generally have smaller corrections than 2.5, but, except for black surfaces, the correction is appreciable and must be made in the low-gloss range in order to grade correctly low-gloss surfaces of different luminous reflectance. To determine the diffuse correction one must take into account the following: (a) reflectance of the specimen, (b) departure of practical surfaces from perfect diffusors, and (c) polarization of the source.

\section{(a) Specimen Reflectance}

With a goniophotometer or other glossmeter in which the illuminator can be rotated about the specimen in the plane of measurement, this correction is most easily evaluated by illuminating the specimen perpendicularly and viewing at an angle of $60^{\circ}$. When this is done the area illuminated is one-half of that previously illuminated, but the flux density is twice as great. If the surface is uniform, the diffuse correction is obtained directly except that a very small error is involved because the surface reflection is less for perpendicular than for $60^{\circ}$ illumination. For a polished specimen having a refractive index of 1.53 , the surface reflectance at $60^{\circ}$ illumination is about 9 percent and at $0^{\circ}$ is about 4 percent. This means that an additional 5 percent is added to the body illuminance, but since this flux is distributed over a hemisphere the increase in light entering the receiver is small. The fraction of the total light entering the receiver was previously computed to be 0.0025 . Since the surface-reflected light is now decreased by 5 percent, the body-reflected light is increased by the same fraction, so that the diffuse correction for $60^{\circ},-60^{\circ}$ condition as measured under $0^{\circ},-60^{\circ}$ conditions is high by 5 percent of the magnitude. This means that for a speci- 
men having a measured diffuse correction of 0.0021 the true diffuse correction would be 0.0020 or, on the usual specular gloss scale, the discrepancy would be 0.1 gloss unit. This discrepancy in the diffuse correction is proportional to the gloss, and is zero for a perfectly diffusing surface. In practice this discrepancy is so small that correction for it is unnecessary. If, on the other hand, $60^{\circ}$ illumination and perpendicular viewing are used, the instrument reading will have to be halved to obtain the diffuse correction, because the projected area for perpendicular view is twice that for $60^{\circ}$ view.

\section{(b) Practical Diffusors}

On the master instrument, figure 4, at this Bureau it was found that magnesium oxide, which is often thought of as the practical approach to the perfectly reflecting, if not perfectly diffusing surface, has a diffuse correction of 2.1. This value was obtained numerous times and though not vastly different from 2.5 certainly gives a larger discrepancy than could be accounted for by experimental error. Previous investigation of the diffusing properties of the surfaces of magnesium oxide by McNicholas [8] and Preston [9] revealed that it is not a perfect diffusor. This was confirmed by us for magnesium oxide; and similar data were obtained for two other materials. Figure 13 illustrates graphically the results of this investigation.

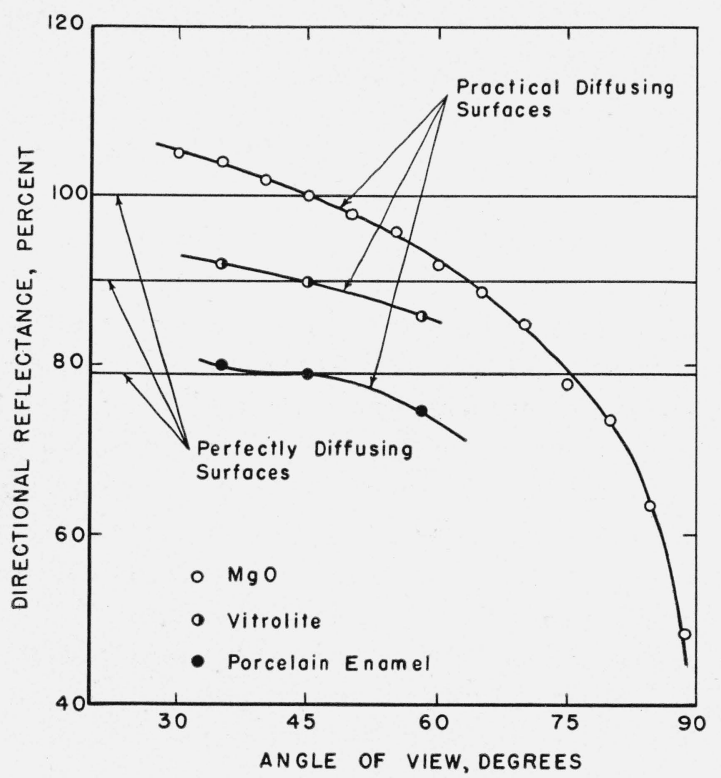

Figure 13. Departure of practical surfaces from the concept of the perfect diffusor.
It will be recalled that the reflectance of magnesium oxide prepared in a prescribed manner [10] is assigned a value of 1.00 when illuminated at $45^{\circ}$ and viewed perpendicularly. For $0^{\circ}, 60^{\circ}$ conditions the directional reflectance of these oxide specimens was found to be only 92.5 percent of the value assigned for $0^{\circ}, 45^{\circ}$ illuminating and viewing conditions. This accounts for nearly half the discrepancy found between the computed and the measured values for the diffuse correction.

\section{(c) Source Polarization}

It will be recalled that the Fresnel reflectance of a surface is a function of the degree of polarization of the incident light as well as the angle of incidence and the index of refraction of the surface. The degree of polarization of the source was measured by rotating a polaroid filter in the emitted beam and recording the maximum and minimum transmittances. The difference of the readings was divided by the sum to obtain polarization. The data obtained show that this source (a C-6 filament) is polarized 5 percent along the axis of the coil, or perpendicular to the plane of measurement. Filament polarizations of 15 to 20 percent for straight filaments were reported by Worthing [11]. Because the $60^{\circ}$ angle is almost the polarizing angle for glass, approximately 5 percent less incident flux is required to obtain the same reflected flux from a polished glass surface than if the source were unpolarized. Although the polarization is destroyed by a low-gloss surface, partial polarization of the incident light causes a greater fraction to be reflected from the polished surface of the high-gloss standard. Consequently, the reduced incident light on the specimen reduces the computed value of the diffuse component by 5 percent of its magnitude.

These three sources of error, (1) surface reflectance, (2) departure from perfect diffusor, and (3) polarization of the source, taken together account for the discrepancy between the computed and the measured values of the diffuse correction.

\section{Gloss Scale and Standards}

Any assembly of apparatus such as a glossmeter requires calibrating and the adoption of a scale on which measurements will be based. The theoretical specular gloss standard is the perfect mirror that is assigned a value of 1,000. The practical 
primary standard is a piece of polished black glass whose specular reflectance is computed from Fresnel's equation when the index of refraction of the glass is known. Black glass is used for this purpose, because all of the light flux not specularly reflected is absorbed by the body of the material and the problems associated with diffuse correction are eliminated.

In order to provide means for checking the adjustment of glossmeters and to assist in more accurate measurement of $60^{\circ}$ specular gloss, this Bureau has investigated the suitability of various materials for use as standards, and has recently assembled sets of standards for issue. Each set consists of two white Vitrolite gloss plaques having nominal gloss values 1 and 90, and eight glazed ceramic plaques having nominal gloss values 10, 20, $30,40,50,60,70$, and 80 . The plaques are approximately $4 \frac{1}{4} 4$ in. square. Figure 14 is a photograph of the plaques together with the box in which the set of ten standards is furnished. Although the plaques are not intended to be used as standards of distinctness-of-image gloss, the photograph in figure 15 has been taken to indicate approximately the gloss steps involved, since for these plaques $60^{\circ}$ specular gloss steps agree in order with distinctness-of-image gloss steps estimates.

\section{Summary and Recommendations}

Although photoelectric measurements of specular gloss at a particular angle are convenient for the evaluation of surface appearance, such measurements do not completely evaluate the gloss characteristics, much less the over-all appearance of the surface. In general, the specular gloss of high-gloss surfaces can best be differentiated by observation at small angles from the perpendicular,

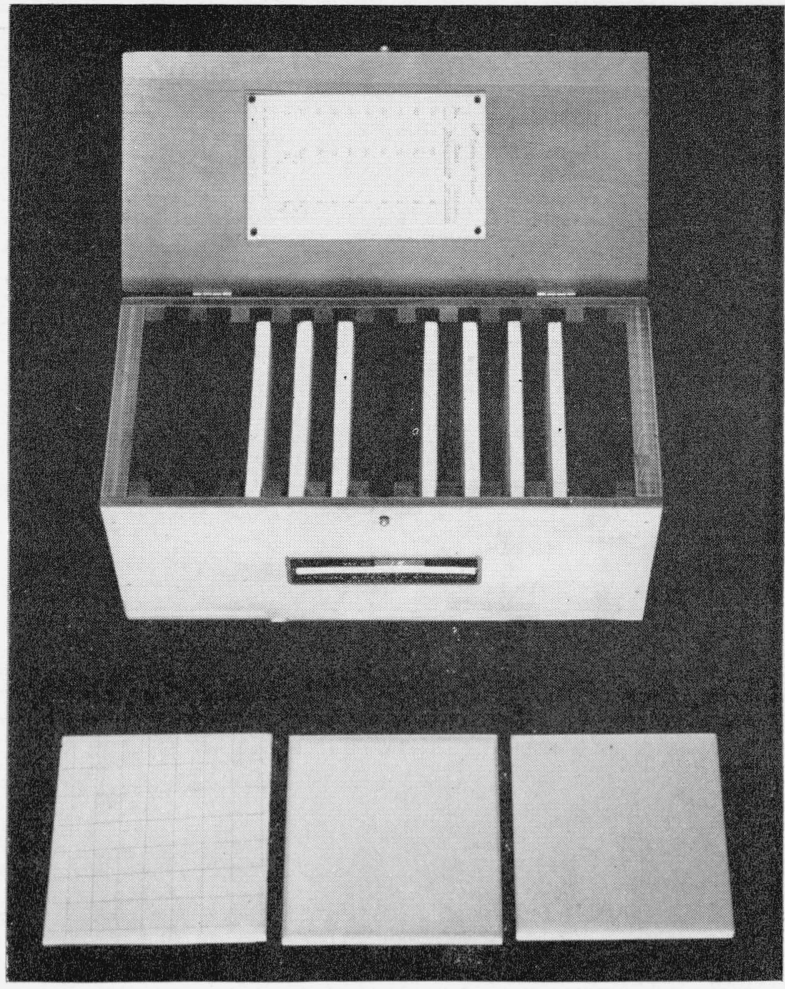

Figure 14. Set of NBS gloss standards, with box in which they are issued.

whereas matte surfaces can best be differentiated at large angles from the perpendicular.

Problems inherent in practical glossmeters have been empirically treated here. After permissible discrepancies in gloss values have been decided upon, the tolerances for the geometric variables for $60^{\circ}$ specular gloss can be obtained from the curves in figures 5 to 9 . If a deviation of \pm 1 gloss unit is specified, then the tolerances can be readily ascertained. On the basis of the data obtained by the authors on the versatile glossmeter and a permis-

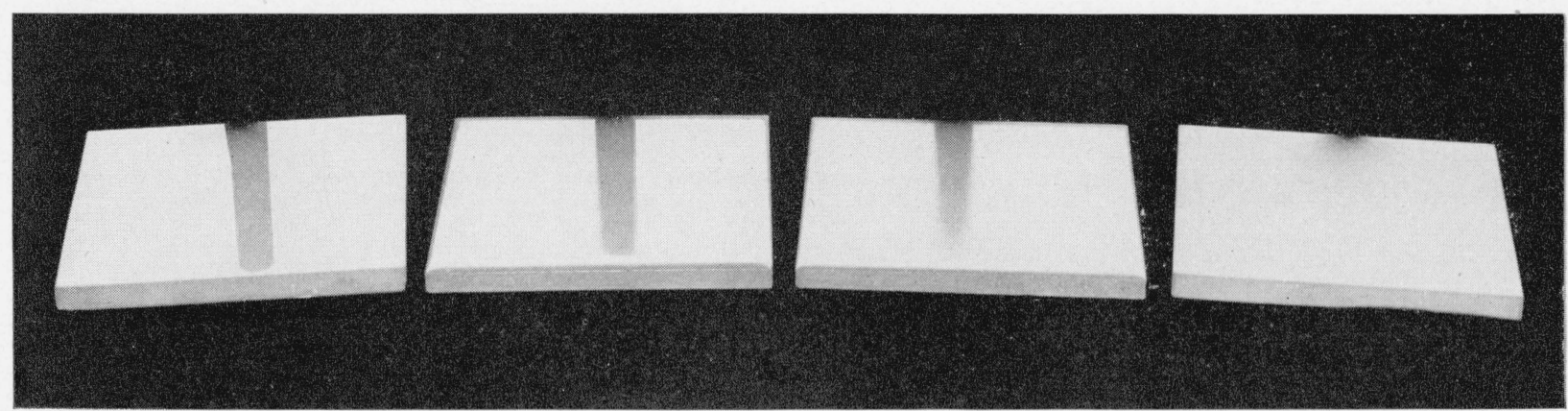

FIGURE 15. Photographic representation of specular gloss standards.

The standards are arranged in order of decreasing specular gloss from left to right, as shown by the more readily photographed gradation of the distinctness of reflected images. For these standards distinctness-of-reflected-image gloss correlates with specular gloss. 
sible deviation of \pm 1 gloss unit, the recommendations shown in table 1 are made.

TABLE 1. Recommended tolerances for practical $60^{\circ}$ glossmeters

\begin{tabular}{|c|c|}
\hline Variable & Recommended tolerance \\
\hline Receiver aperture: & \\
\hline In plane of measurement & $\pm 0.1^{\circ}$. \\
\hline $\begin{array}{l}\text { Perpendicular to plane of meas- } \\
\text { urement. }\end{array}$ & $\pm 0.2^{\circ}$ \\
\hline $\begin{array}{l}\text { Source aperture in plane of meas- } \\
\text { urement. }\end{array}$ & $\pm 0.2^{\circ}$ \\
\hline Position of source image ${ }^{1} \ldots$ & $\begin{array}{l} \pm 5 \% \text { of the focal length of re- } \\
\text { ceiver lens. }\end{array}$ \\
\hline Specular angle... & $\begin{array}{l} \pm 0.3^{\circ} \text { from the specified spec- } \\
\text { ular angle. }\end{array}$ \\
\hline
\end{tabular}

${ }^{1}$ For uncorrected lenses, source image should be focussed for the circle of least confusion.

In view of the fact that these discrepancies are cumulative, it is recommended that manufacturers and users of practical glossmeters be careful that the geometric variables be held well within the recommended tolerances; for if all the variables happen to contribute errors in the same direction though they are just within the recommended tolerances, the reading may be in error by as much as 5 gloss units.

The authors express their appreciation to D. B. Judd for invaluable suggestions and advice during preparation of this paper and also to E. M. Hagaman for his ingenuity and assistance in the construction of the versatile glossmeter.

\section{References}

[1] R. S. Hunter and D. B. Judd, ASTM Bulletin No. 97, 11 (March 1939).

[2] A. Fresnel, Calcul des teintes que polarization developpe dans lames cristallisees, Ann. chim. phys, 17, 194, 312 (1821).

[3] R. S. Hunter, Methods of determining gloss, J. Research NBS 18, 19 (1937).

[4] ASTM tentative method of test for $60^{\circ}$ specular gloss of paint finishes, ASTM designation D523, Book of ASTM Standards, Part II (1949).

[5] S. C. Horning and M. P. Morse, The measurement of the gloss of paint panels, Official Digest (Fed. Paint and Varnish Products Clubs) 266, 153 (1947).

[6] D. G. Moore and R. S. Hunter, Use of liquid surfaces as standards of specular gloss, J. Am. Ceramic Soc. 24, 167 (1941).

[7] J. H. Lambert, Photometria sive de mensura et gradibus luminis, colorum et umbrae, p. 324 , Augsburg (1760).

[8] H. J. McNicholas, Absolute methods in reflectometry, BS J. Research 1, 29 (1928) RP3.

[9] J. S. Preston, The reflection factor of magnesium oxide, Trans. Opt. Soc. (London) 31, 15 (1929).

[10] NBS Letter Circular LC547, Preparation and colorimetric properties of magnesium-oxide reflectance standard (1939).

[11] A. G. Worthing, Deviation from Lambert's law, and polarization of light emitted by incandescent tungsten, tantalum, and molybdenum, and changes in the optical constants of tungsten with temperature, J. Opt. Soc. Am. 13, 635 (1926).

Washington, February 13, 1950. 